\title{
Analytical kinematics and trajectory planning of large scale hexagonal modular mesh deployable antenna
}

\author{
Mariyam Sattar and Cheng Wei \\ School of Aeronautics Science \& Engineering, Beihang University, Beijing, 100191, Haidian District, China
}

\begin{abstract}
This investigation aims to analyze the forward kinematics and deployment path of large scale hexagonal modular mesh deployable space antenna employing analytical procedure. At first a comprehensive review of technical contributions from 1987 to date in this class of antenna is completed and least targeted areas are extracted. Based on shortcomings a new design of modular antenna is proposed that has an aperture diameter of 13 meters and is composed of seven modules. Each module possesses an aperture of more than 6 meters and consists of 6 ribs arranged in radial pattern around the central beam. In order to ensure that the antenna will deploy successfully in space conditions, feasibility of design \& its stability during deployment; analytical investigation into kinematics \& antenna trajectory is carried out using loop closure equations and homogeneous coordinate transformation method. The concept of virtual link is introduced to develop formulations that will help to determine position, velocity and acceleration of each revolute joint in the rib with any small increment in linear motion of slider from top to bottom of central beam. The proposed solution techniques will help to replace time consuming and costly iterations for design, build more economical structure, reduce antenna manufacture time and reduce weight leading to reduced launch as well as experimentation costs before launch.
\end{abstract}

\section{Introduction}

Space deployable antenna is an essential aerospace equipment to land and space exploration activities [1], [2]. In past, extensive researches about antenna design have taken place ranging from highly accurate but heavy solid reflectors [3] to extremely light inflatable ones. In between these two extremes are the mesh antenna [4]. Hexagonal module antenna, with high stiffness, packaging efficiency \& high accuracy of observation is one of its subcategories. The shape of this reflector is maintained by mesh surface supported by cable network and deployable truss. The integrity of support structure is the most important factor that needs to be confirmed before final launch. Current expedition is to research \& apply some cost effective \& efficient methods of analysis to minimize the risk of failure and confirm stability of design during launch or in-orbit operation.

\section{Background Study}

Nippon Telegraph and Telephone Corporation has been developing modular mesh deployable antenna since 1987 [5]-[7] for S-band mobile communication satellites. Since then methods for, basic module unit design, their integration \& alignment, mesh \& cable scrutiny, surface accuracy analysis, the deployment mechanism, in-orbit \& ground modularized testing, analytical \& simulation tools, have been developed to verify and maximize the performance of antenna. Initially, multi-beam, mechanical \& measurement technologies were used to construct partially deployable hexa-link truss and tetra trigonal prism [8]. Later, a variety of basic modular units, such as square grid deployable [9]-[11], two types of basic modules with one being able to stow as cylinder \& the other to thin plate form [12], [13], quadrilateral ribs with diagonal members for ETS VIII antenna [14]-[33], tri-foldable ribs [34], [35] and tri-foldable with bifurcated rib tips [36] for L-band application, have been proposed to integrate hexagonal reflector with aperture ranging from $1.7 \mathrm{~m}$ to $30 \mathrm{~m}$ in diameter. In [27], [29] six different rib schemes based on ETS VIII antenna units are discussed and fuzzy comprehensive evaluation method is applied [29] to determine the optimized configuration. Quest to increase aperture and reduce weight has led to design 13m, 14 modules ETS VIII antenna of around $340 \mathrm{~kg}$ [15], [17] , 30m, 7 modular antenna composed of tri-foldable 4 sided ribs of $250 \mathrm{~kg}$ [34], [35] and recently $15 \mathrm{~m}$ aperture antenna of $58 \mathrm{~kg}$ composed of tri-foldable bifurcated rib tips [36]. However, there is an increase in level of complexity of deployment mechanism with introduction of tri-foldable ribs in design. When modules are connected to each other displacement issue at connection points arise leading to shape and alignment errors. Harada et. al, [37] proposed an error approximation method to estimate variation of structural displacements at end points by deriving the covariance matrix for the displacement vector, while least square [24], correction by varying angles between ribs in the 
modules \& equal envelop circle radius method [27] are employed to adjust alignment error. Meguro quantitatively assessed influence of alignment errors on surface accuracy and proposed to adjust tie cable length to improve it [10]. It is suggested [20] to use extremely stiff surface cables \& relatively elastic ones as back \& supporting cables to reduce deformation sensitivity \& achieve high surface accuracy of antenna. Surface accuracy effected by thermal deformation in orbit is actively compensated by using shape measuring sensors [38]. Numerous numerical \& analytical procedures are applied to refine parameters of modular antenna. Numerical procedures include MSC/NASTRAN [14], OOCD [12], [13], [32] \& Origami/ETS [36] for design \& structural analysis, CASA [9]-[11] for analysis of reaction forces \& moments created by mesh \& cable network, DADS [9]-[13] for deployment motion analysis, NEVADA/SINDA for thermal analysis [14], SPADE for flexibility analysis of truss [12], [13], [32], [39]-[41] \& Flexible Multi-body dynamics simulation [14, 16, 42] to determine deployment driving force. Mitsugi analytically measured cable strains by integrating stiffness matrix of the support structure into nonlinear cable analysis equation to cope with elastic deformation [11]. In [43] strain induced in cable element during deployment are determined. Ando [39] used direct coordinate partitioning method to reduce DoF of joints contributing to design of deployable truss. Later, homogeneous coordinate transformation method is used for kinematics [30] \& geometric modeling [24] of deployable truss with each module sharing the same geometric parameters. In [28] kinematics of rib unit is investigated by dividing it into three one DoF 4-link sub-structures. Misawa predicted [44] and verified [45] natural frequencies for stiffness design of deployed antenna. Later, in [31] multi-body dynamics theory is employed to determine rigid body dynamics of a rib. In [25], [26], [28] simulations on deployed antenna are worked in ANSYS to obtain free, harmonic \& damped transient dynamic response in terms of natural frequencies \& modes. Another targeted section is the influence of chord, beams \& cables diameter [26, 28] and flexibility of linkages [31] on the dynamic properties. Stiffness \& frequency can be enhanced by improving diameter of chord and diagonal beam. While flexibility that led to bending and fluctuation of velocity $\&$ acceleration can be controlled by using cell bodies of higher density, elastic modulus \& cross-sectional area. Many ground experimental tests [9], [12], [13], [15]-[19], [21]-[23], [33], [40], [41], [43], [46], [47] are conducted to verify design criteria $[9,12]$, analysis method [12], [16], [19], [43], deployment reliability [13], [17], [18], [21] and in-orbit operation performance of antenna [23]. Ground deployment tests equipment mostly consisted of suspension wires, pulleys \& counter weights, to overcome gravity [12], [13] however, in [46] a vertical support using a magnetically suspended slider system is proposed to create $0 \mathrm{G}$ conditions. Meguro considered items like elastic deformation, contingency mode and mesh fold pattern for more realistic analysis of deployment [18]. Moreover, deployment testing on ground is more accurate if it is performed on a single module [40]. In ground deployment testing gravity interference highly affects the analysis results as in [9] unexpected loads arose in the hinge mechanisms. Doubtlessly, because of limited gravity compensation, gravity torque is induced that affect and change deployment characteristics. Despite the amount of extensive research into deployable structures, several high-profile failures have occurred. Failures in space are very expensive \& extremely difficult to correct. The robustness of the concept has to be first proven by analytical models. Extraction from background study depicts that mostly simulations; ground and in-orbit experimental tests are completed to analyze the performance characteristics of modular antenna. Limited analytical procedures either on single rib of truss module or for one module is available to determine the kinematics while for dynamics and static topology optimization there is lack of any such technique. Here attempts are made to develop analytical methods that may assure stability and kinematic performance of large modular antenna prior to operation by checking the deployment trajectory. In the proceeding section, antenna design \& problem description is conversed; formulation for kinematics and trajectory planning is obtained next, followed by conclusion and future recommendations.

\section{Antenna details and problem description}

Deployable reflector antenna under consideration consists of mesh surface, cable network and deployable truss structure. Mesh surface is made of gold-plated molybdenum or beryllium copper wire. Cable network consists of surface, tie and back cables. The mesh \& cable network are attached to the rigid structure by stand-offs and tension cables. Rigid support is a deployable truss consisting of several basic modules. Each module is a hexagonal truncated pyramid composed of six basic ribs arranged in radial pattern around central beam. Basic rib is a deployable quadrilateral linkage with a diagonal member. Slider on central beam initiates and control deployment of ribs with the help of motor that releases the restrictive cables uniformly to avoid impact at the end of deployment. Deployable truss is designed to be spherical shape because all modules have almost the same design so, they are easy to handle and adjust the parabolic shape of mesh surface. In addition the number of modules can be conveniently varied to construct different antenna aperture sizes.

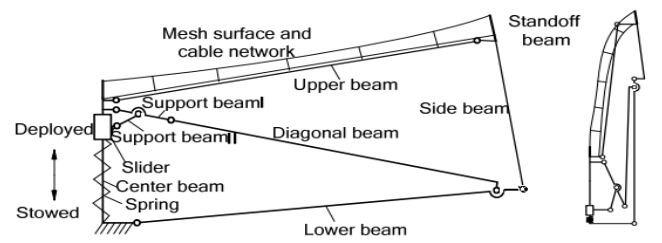

Fig. 1 Basic quadrilateral rib with diagonal member [14].

In present work, the antenna structure consists of 7 modules with each of it more than $6 \mathrm{~m}$ in diameter contributing to give an aperture diameter of $13 \mathrm{~m}$. Basic rib shown in Fig. 1 is used as test specimen for initial development of analytical kinematics that will be 
extended to one complete module then to seven modules truss. Analysis is based on following assumptions;

(1) Central beam is fixed and the reference configuration

(2) Rib is a planar \& one DoF structure

\subsection{Kinematics analysis}

To design truss structure of 7 modular antenna and ensure that it will be deployed successfully in orbit forward kinematics analysis is carried out. Kinematics of basic rib is determined at the first step. As basic rib is a one DoF mechanism so, slider driven by spring moves up \& down to complete synchronous deployment and retraction of six ribs in a module. In [28] basic rib is divided into three 1DoF four link substructures (SS). SS-1 consists of slider, support beam (SB) I, central beam (CB) \& SB-II. SS-II consists of SB-I, CB, diagonal and lower beam while third SS has upper, lower, side and CB. CB is assumed to be fixed to determine loop closure equations of all SS. Given the length of the links in the loop closure equations the corresponding angles of the revolute joints are calculated by the following equations;

From SS-I, the angular dimensions are calculate to be;

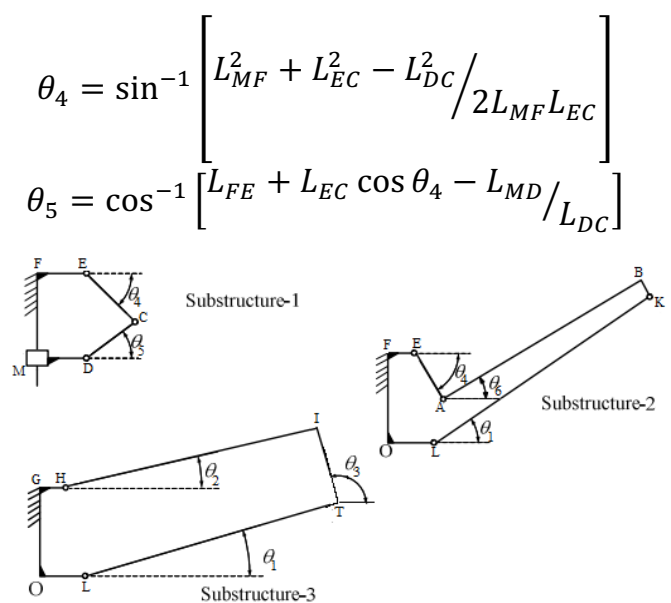

Fig. 2. Substructures of basic rib [28]

From SS-II;

$$
\theta_{6}=\left[\sin ^{-1}\left(L_{1} / \sqrt{P^{2}+Q^{2}}\right)-Z\right]
$$

where; $\mathrm{L}_{1}, \mathrm{P}, \mathrm{Q} \& \mathrm{Z}$ are given by;

$$
\begin{gathered}
L_{1}=L_{L K} \cos \theta_{1}+L_{O L}-L_{F E}-L_{E A} \cos \theta_{4} \\
P=L_{A B} \\
Q=L_{B K} \\
Z=\tan ^{-1}(Q / P)
\end{gathered}
$$

Angle $\theta_{1}$ is calculated to be;

$$
\theta_{1}=\left[\sin ^{-1}\left(L_{2} / \sqrt{R^{2}+S^{2}}\right)-Y\right]
$$

where; $L_{2}, \mathrm{R}, \mathrm{S}, \mathrm{Y}$ are given by;

$$
\begin{gathered}
L_{2}=L_{L K}^{2}+L_{a}^{2}+L_{b}^{2}-L_{A B}^{2}-L_{B K}^{2} \\
R=2 L_{a} L_{L K} \\
S=2 L_{b} L_{L K} \\
Y=\tan ^{-1}\left(S /_{R}\right)
\end{gathered}
$$

$L_{a} \& L_{b}$ will be calculated using following formula,

$$
\begin{gathered}
L_{a}=L_{O L}-L_{F E}-L_{E A} \cos \theta_{4} \\
L_{b}=L_{E A} \sin \theta_{4}-L_{O F}
\end{gathered}
$$

Angles in SS-III are given by,

$$
\theta_{2}=T+\theta_{3}
$$

where; ' $\mathrm{T}$ ' in the above equation is;

$$
\begin{gathered}
T=\cos ^{-1}\left[L_{H J}^{2}+L_{J K}^{2}-L_{c}^{2}-L_{d}^{2} / 2 L_{H J} L_{J K}\right] \\
L_{c}=L_{O L}+L_{L K} \cos \theta_{1}-L_{G H} \\
L_{d}=L_{L K} \sin \theta_{1}-L_{O G}
\end{gathered}
$$

For $\theta_{3}$;

$$
\begin{gathered}
\theta_{3}=\left[\sin ^{-1}\left(L_{3} / \sqrt{U^{2}+V^{2}}\right)-X\right] \\
L_{3}=L_{L K} \sin \theta_{1}-L_{O G} \\
U=L_{H J} \times \sin T \\
V=\left(L_{H J} \times \cos T\right)-L_{J K} \\
X=\tan ^{-1} V / U
\end{gathered}
$$

The displacement of slider in SS-I is an input variable handling deployment and stowage by varying the six angular dimensions i.e. $\Theta_{1}, \Theta_{2}, \Theta_{3}, \Theta_{4}, \Theta_{5} \& \Theta_{6}$.

In order to determine the positions and hence the trajectory of connection point ' $\mathrm{K}$ ' with motion of slider from top to bottom; virtual link between point ' $M$ ' and ' $L$ ' is considered. $\mathrm{CB}$ and link ' $\mathrm{OL}$ ' will always maintain an angle of $90^{\circ}$. From geometry;

$$
L_{M L}=\sqrt{L_{O M}^{2}+L_{O L}^{2}}
$$

where;

$$
\alpha=\cos ^{-1} L_{O L} / L_{M L}
$$

So, the position of revolute at point ' $\mathrm{K}$ ' with every increment in distance between point ' $\mathrm{M}$ ' \& ' $\mathrm{F}$ ' is;

$$
\begin{gathered}
x_{K}=L_{O L}+L_{L K} \cos \theta_{1} \\
\text { Similarly, } \begin{array}{l}
x_{K}=L_{M L} \cos \alpha+L_{L K} \cos \theta_{1} \\
y_{K}=L_{M L} \sin \alpha+L_{L K} \sin \theta_{1}
\end{array}
\end{gathered}
$$

So, any change in length between points ' $M$ ' and ' $F$ ' will cause variation in distance between origin and point $\mathrm{M}$. This will lead to change of length of link ' $\mathrm{ML}$ ' and the angle it forms with the $\mathrm{x}$-axis thus, changing the position ' $\mathrm{K}$ ' of the revolute on lower beam with each millimeter variation in position of slider ' $M$ '. As rib is one DoF mechanism so, position of any revolute on the rib with respect to translational motion of slider can be calculated in the same manner by introducing virtual link.

Now in order to extend the kinematics from basic rib to complete module the method using combination of homogeneous coordinates \& transformation matrix is applied. A coordinate system is established at the bottom of central beam. $\mathrm{X}$-axis is from origin to positive $\mathrm{x}$ direction, $\mathrm{y}$-axis is from bottom of center beam towards the top i.e. positive $y$-direction and $z$-axis points towards the reader. There are six ribs in each module. Consider the one under consideration as rib 1. Homogeneous coordinates of revolute at point ' $\mathrm{K}$ ' on the bottom link of rib 1 are given by;

$$
K_{1}=\left[\begin{array}{llll}
x_{k_{1}} & y_{k_{1}} & 0 & 1
\end{array}\right]^{T}
$$


Six ribs are spaced at an equal angle of ${ }^{\prime} \pi / 3{ }^{\prime}$ around the central beam. Transformation matrix to determine location of each rib in the module is actually the rotation by an angle ' $\beta_{n}$ ' around the y-axis and is given by;

$$
\operatorname{Rot}\left(y, \beta_{n}\right)=\left[\begin{array}{cccc}
\cos \beta_{n} & 0 & \sin \beta_{n} & 0 \\
0 & 1 & 0 & 0 \\
-\sin \beta_{n} & 0 & \cos \beta_{n} & 0 \\
0 & 0 & 0 & 1
\end{array}\right]
$$

where, ' $\beta_{n}$ ' is given by ' $n \pi / 3$ '; $\& \mathrm{n}=0,1,2,3,4,5$ for one complete module. So, the homogeneous coordinates of any connection point in rib under consideration can be given by;

$$
\begin{aligned}
\mathrm{H}_{i}=\operatorname{Rot}\left(y, \beta_{n}\right) & \times \mathrm{H}_{1} \\
& =\left[\begin{array}{cccc}
\cos \beta_{n} & 0 & \sin \beta_{n} & 0 \\
0 & 1 & 0 & 0 \\
-\sin \beta_{n} & 0 & \cos \beta_{n} & 0 \\
0 & 0 & 0 & 1
\end{array}\right] \times\left[\begin{array}{c}
x_{\mathrm{H}_{1}} \\
y_{\mathrm{H}_{1}} \\
0 \\
1
\end{array}\right] \\
& =\left[\begin{array}{c}
x_{\mathrm{H}_{1}} \times \cos \beta_{n} \\
y_{\mathrm{H}_{1}} \\
-x_{\mathrm{H}_{1}} \times \sin \beta_{n} \\
1
\end{array}\right]
\end{aligned}
$$

where ' $\mathrm{H}$ ' will change from 'A to $\mathrm{M}$ ' \& ' $i$ ' will modify its value from ' 1 to 6 ', in fact the general formulation for ' $i$ ' is $i=n+1$. So, the general model of deployment kinematic analysis giving the formulation to determine the position of any point on the structure has been developed. Provided the length of links this will help to analyze deployment process and trajectory of revolutes on the structure with change of position of slider. The nodal velocities and accelerations can be determined by taking the first and second derivative of nodal displacement equation with respect to time. The same procedure of transformation matrix will be extended to 7 modular antenna to determine kinematics and trajectory of connection points in rest of modules with respect to basic coordinate system.

\section{Conclusion and future recommendations}

Large deployable hexagonal modular mesh antenna is research object in this paper. A comprehensive review of technical contributions from 1987 to date in this class of antenna is completed and least targeted areas are extracted. Based on shortcomings a new design of a 7 modular deployable antenna of $13 \mathrm{~m}$ aperture is proposed. Each module in design is more than $6 \mathrm{~m}$ in diameter. In order to ensure that the antenna will deploy successfully in space conditions, analytical investigation into kinematics is carried out using loop closure equations and homogeneous coordinate transformation method. In order to ensure feasibility of design \& its stability during deployment process formulations are developed to determine antenna trajectory.

In order to validate analytical kinematics, design of truss structure composed of 7 modules in Solid works is underway. The geometry will be imported to ADAMS software to work out deployment process and trajectory of overlapping points of modular structure that will help to authenticate analytical kinematics. The deployment sequence of the structure will be like the one depicted in Fig. 3.
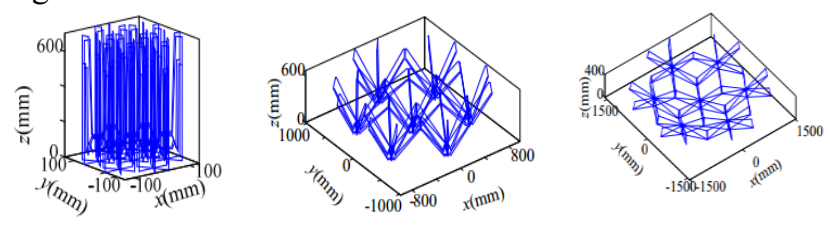

Fig. 3. Deployment sequence of deployable truss [28]

Previous researchers have used a module of $4.8 \mathrm{~m}$ aperture dia. to design $13 \mathrm{~m} \& 9.56 \mathrm{~m}$ aperture antenna by combining $14 \& 7$ modules respectively. The highest value of fundamental natural frequency achieved was $0.14 \mathrm{~Hz}$ [17] for 14 module antenna while for a 7 modular it is $0.25 \mathrm{~Hz}$ [48]. It is found that using 14 modules contribute to an increase in weight at the cost of stiffness and natural frequency. So, authors are working on 7 integrated modules to design $13 \mathrm{~m}$ aperture antenna that will improve stiffness, reduce fabrication time, cost \& complexity of design and will provide long distance and large scale communication with reduced number of modules. Its expected value of fundamental frequency will be between $0.14 \mathrm{~Hz}$ and $0.25 \mathrm{~Hz}$. Analytical \& simulation procedures will be attempted to verify these results. Analytical coupled dynamic model, incorporating the influence of thermal \& $0 \mathrm{G}$ conditions, of deployable truss will be developed using Lagrange method to obtain response in terms of natural frequency. Positions \& velocities obtained from kinematic model will be used to calculate potential \& kinetic energies of the system to determine the Lagrangian which will further be solved for frequency response. The results will be validated by comparing the results with FE simulations run in ANSYS to get natural frequencies and the features of vibration modes. Deployable antenna is very large and their natural frequency is usually very low. Structures with frequencies falling in low frequency regime are more likely to observe resonance. So in order to avoid resonance deployable antenna is designed after setting some requirements in which main focus is to increase stiffness and reduce mass to acquire frequency response. Based on the natural frequency response of antenna improvements can be applied to the design to get optimized performance. Moreover the study of natural frequency response is important because it gives modal analysis to study the dynamics of vibrations, accuracy of design and stability of antenna.

Optimization can be done to replace time consuming iterations for design, build more economical structure, reduce antenna manufacture time and reduce weight leading to reduced launch $\&$ experimentation costs before launch. Authors intend to use Solid Isotropic Material with Penalization (SIMP) method to investigate the topology optimization of the structure. The objective function will be to maximize the value of stiffness and frequency response with least number of modules for desired mission application.

\section{References}


1. Angeletti P., M. Lisi, and G. Lucchi, $2^{1 s t}$ International Communications Satellite Systems Conference and Exhibit, Yokohama, Japan, (2003).

2. Mini F., et al., The Second European Conference on Antennas and Propagation, EuCAP, IET, (2007) .

3. Medzmariashvili E., et al., Proceedings of Earth \& Space, (2006).

4. Gunnar T., Deployable tensegrity structures for space applications. Ph. D. Dissertation, (2002): p. 13-31.

5. Mitsugi J. and T. Yasaka, Space Technology and Science, (1990).

6. Mitsugi J. and T. Yasaka, IAF, 42 ${ }^{\text {nd }}$ International Astronautical Congress, Montreal, Canada, (1991).

7. Meguro A., et al., $20^{\text {th }}$ International Symposium on Space Technology and Science, Gifu, Japan, (1996).

8. Ebisui T., et al., $14^{\text {th }}$ AIAA International Communication Satellite Systems Conference and Exhibit, Washington DC, (1992).

9. Meguro A., NASA Conference Publication, (1993).

10. Meguro A., J. Mitsugi, and K. Ando, Electronics and Communications in Japan (Part I: Communications), (1994). 77(8): p. 90-100.

11. Mitsugi J., Computers \& structures, (1994). 51(1): p. 47-56.

12. Mitsugi J., Proc. Sixth European Space Mechanisms and Tribology Symposium (1995), Technopark: Zurich, Switzerland.

13. Watanabe M., et al., Acta Astronautica, (1996). 39(7): p. 497-505.

14. Nakamura K., et al., $18^{\text {th }}$ ICSSC, AIAA-98-1229, (1998).

15. Meguro A., A. Tsujihata and N. Hamamoto, Antennas and Propagation Society International Symposium, (1999), IEEE.

16. Mitsugi J., et al., Acta Astronautica, (2000). 47(1): p. 19-26.

17. Meguro A., et al., Acta Astronautica, (2000). 47(2): p. 147-152.

18. Meguro A., et al., $42^{\text {nd }} A I A A / A S M E / A S C E / A H S / A S C$ Structures, Structural Dynamics and Material Conference and Exhibit, (2000).

19. Miyasaka A., M. Hommat, and A. Tsujigata, $42^{\text {th }}$ MAA/ASME/ASCE/AHS/ASC Structures, Structural Dynamics, and Materials Conference and Exhibit. Seattle, WA, United states: MAA, (2001).

20. Harada S., A. Meguro, and M. Watanabe, $44^{\text {th }}$ AIAA/ASME/ASCE/AHS/ASC Structures, Structural Dynamics, and Materials Conference, (2003).

21. Yamada K., et al., International Communications Satellite Systems Conference and Exhibit, Yokohama, Japan, (2003).

22. Meguro A., H. Ishikawa and A. Tsujihata, J Spacecr Rockets,( 2006). 43(4): p. 780-787.

23. Meguro A., et al., Acta Astronautica, (2009). 65(9): p. 1306-1316.

24. Tian D., R. Liu, and Z. Deng, International Conference on Measuring Technology and Mechatronics Automation (ICMTMA), IEEE, (2010).

25. Tian D.K., et al., Applied Mechanics and Materials, Trans Tech Publ, (2010).
26. Tian D., et al. $9^{\text {th }}$ World Congress on Intelligent Control and Automation (WCICA), IEEE, (2011).

27. Fei C., Chinese Journal of Aeronautics, (2009).

28. Guo H., et al. 6th European Conference on Antennas and Propagation (EUCAP), IEEE, (2012).

29. Tian D., et al., Applied Informatics and Communication (2011), Springer. p. 178-185.

30. Liu R.Q., et al., Advanced Materials Research, Trans Tech Publ, (2012).

31. Wang S.M., S. Chen, and R. Yuan, Applied Mechanics and Materials, Trans Tech Publ, (2013).

32. Meguro A., S. Harada, and M. Watanabe, Acta Astronautica, (2003). 53(11): p. 899-908.

33. Ishikawa H., A. Meguro, and M. Watanabe, Transactions of the Japan Society for Aeronautical and Space Sciences, (2004). 47(157): p. 175-180.

34. Ozawa S., K. Shintate, and A. Tsujihata. Proceedings of the $5^{\text {th }}$ European Conference on Antennas and Propagation (EUCAP), IEEE, (2011).

35. Ozawa S., K. Shintate and A. Tsujihata. Proceedings of the $5^{\text {th }}$ European Conference on Antennas and Propagation (EUCAP), (2011).

36. Nakamura K., et al., $3^{\text {rd }}$ AIAA Spacecraft Structures Conference, (2016).

37. Harada S., M. Shimizu, and T. Mitsugi, AIAA-98, (1998).

38. Ueba M., et al. $15^{\text {th }}$ AIAA International Communications Satellite Systems Conference, San Diego, CA. (1994).

39. Ando K., J. Mitsugi and Y. Senbokuya, Computers \& structures, (2000). 74(1): p. 21-39.

40. Meguro A., H. Ishikawa, and A. Tsujihata, Proceedings of the $43^{\text {rd }}$ AIAA/ASME/ASCE/AHS/ASC Structure, Structural Dynamics and Material Conference, (2002).

41. Tsujihata A., et al., Large Deployable Reflector (LDR) onboard Engineering Test Satellite (ETS-VIII), (1998).

42. Mitsugi J. Proceedings of $36^{\text {th }}$ Structures, Structural Dynamics, and Material Conference, AIAA, (1995).

43. Mitsugi J. and Y., Proceedings of the $37^{\text {th }}$ Structural Dynamics, and Materials Conference, AIAA, (1996).

44. Misawa M. and J. Mizuno, A Collection of Technical Papers: AIAA/ASME/ASCE/AHS/ASC Structures, Structural Dynamics and Materials Conference and Exhibit, AIAA/ASME/AHS Adaptive Structures Forum, AIAA Non-Deterministic Approaches Forum, AIAA Gossamer Spacecraft Forum: Seattle, Washington, 16-19 April, (2001).

45. Misawa M. and A. Ogawa, Analytical and experimental frequency verification of deployed satellite antenna, (2003). 2: p. 3.

46. Sugimoto T., et al.,38 ${ }^{\text {th }}$ Structures, Structural Dynamics, and Materials Conference, (1997).

47. Nagaoka N., et al., $44^{\text {th }}$ AIAA / ASME / ASCE / AHS / ASC Structures, Structural Dynamics, and Materials Conference, (2003).

48. Higuchi K., et al., Proc. 50 ${ }^{\text {th }}$ AIAA / ASME / ASCE / AHS / ASC Structures, Structural Dynamics and Materials Conference, AIAA-2009-2609, (2009). 